\title{
Fenómenos de exclusión social en asentamientos precarios: desafíos para las políticas públicas y las organizaciones de la sociedad civil, OSC
}

\author{
Carla Fierro ${ }^{1}$, Carla Martínez ${ }^{2}$, Andrés Estay ${ }^{3}$ y \\ Emanuel Bustos ${ }^{4}$
}

\section{RESUMEN}

Las investigaciones actuales sobre los fenómenos asociados a la exclusión social, tales como las migraciones, tienen relación con la visibilidad de los asentamientos precarios como parte esencial de la desigualdad territorial y segregación social en Chile; sin embargo, aún persisten desafíos importantes para su adecuada caracterización cualitativa y respecto de cómo los diversos fenómenos de exclusión social detectados en asentamientos precarios en Chile desafían a las políticas públicas y las organizaciones de la sociedad civil, y por tanto a la intervención social que busca superarlos.

Este artículo busca contribuir a esta discusión y poner en relieve las consecuencias y análisis de estas subexclusiones sociales en asentamientos precarios, a partir de revisión bibliográfica, análisis de datos secundarios y estudio de casos.

Palabras clave: asentamientos precarios, exclusiones sociales, intervenciones sociales y políticas públicas

\section{Phenomena of Social Exclusion in Precarious Settlements: Challenges for Public Policies and CSOs}

ABSTRACT

Current research on phenomena associated with social exclusion, such as migration, are related to the visibility of precarious settlements as an

1 Chilena. Fundación Quiero Mi Casa. E-mail: carlafierror@gmail.com

2 Chilena. Fundación Quiero Mi Casa.E-mail: carla.martinezn@mail.udp.cl

3 Chileno. Fundación Quiero Mi Casa. E-mail: andresestaypoblete@gmai.com

4 Chileno. Fundación Quiero Mi Casa. E-mail: emanuelbustosp@gmail.com 
Fenómenos de exclusión social en asentamientos precarios: desafíos para las políticas públicas y las organizaciones de la sociedad civil, OSC / Fierro et al.

essential part of Chile's territorial inequality and social segregation. However, important challenges still remain for its adequate qualitative characterization and to understand how the different social exclusion phenomena detected in precarious settlements in Chile challenge public policy and civil society organizations, as well as the social intervention that seeks to overcome them. This article looks to contribute to this discussion and highlight the consequences and analysis of these social sub-exclusions in precarious settlements, based on a literature review, secondary data analysis and case study.

Keywords: precarious settlements, social exclusion, social intervention, public policy

\title{
Fenômenos de exclusão social em assentamentos precários: desafios para as políticas públicas e as organizações da sociedade civil, OSC
}

RESUMO

\begin{abstract}
As investigações atuais sobre os fenômenos associados à exclusão social, tais como as migrações, apresentam relação com a visibilidade dos assentamentos precários como parte essencial da desigualdade territorial e segregação social no Chile; porém, ainda persistem desafios importantes para sua adequada caracterização qualitativa e como os diversos fenômenos de exclusão social detectados em assentamentos precários no Chile desafiam as políticas públicas e as organizações da sociedade civil e, portanto, a intervenção social que procura superá-los.

Este artigo busca contribuir para esta discussão e colocar em evidência as consequências e análises destas subexclusões sociais em assentamentos precários, a partir da revisão bibliográfica, análise de dados secundários e estudo de casos.
\end{abstract}

Palavras-chave: assentamentos precários, exclusões sociais, intervenções sociais e políticas públicas

\section{Introducción}

Si bien "exclusión social" es un concepto que destaca por su dinamismo y por las variadas dimensiones con las cuales puede asociarse, destacan para la presente discusión las vertientes teóricas que originan el uso del concepto a cómo lo entendemos hoy, pues el correlato entre estos enfoques adscribe al análisis y utilización de este concepto como el fenómeno (o multifenómeno) que permite la explicación de la situación de vulnerabilidad y la descomposición del tejido social de los individuos o grupos 
de individuos que se encuentran bajo esta condición. El caso de los asentamientos precarios reviste gran importancia para el siguiente artículo pues, pese a los intentos que puedan generarse desde el conocimiento, la intervención social y la política pública para hacer frente a los retos que impone la exclusión social en este tipo de contextos, la tarea por asegurar el acceso a oportunidades, garantizar derechos fundamentales mínimos que aumenten su calidad de vida y propiciar la integración efectiva de estas personas al sistema social, se configura como desafío aún pendiente para Chile, en vista y considerando los acelerados procesos políticos, económicos, sociales, culturales y tecnológicos que envuelven a la sociedad contemporánea.

En este sentido, en un primer apartado, se hace un desarrollo del concepto de "exclusión social" a partir de las propuestas teóricas de diversos autores, para, posteriormente, vincularlo con los conceptos de "pobreza" y "vulnerabilidad". En segundo lugar, se asocia el fenómeno de la exclusión social en asentamientos precarios, identificando aquellos factores detectados en estos espacios que se encuentran vinculados a la exclusión, como la migración y racialización, para determinar cómo, desde el enfoque de la intervención social, es posible develar mecanismos facilitadores u obstaculizadores que operen en el nivel de la integración social de estas personas, y cómo, desde la política pública, se ha intentado dar respuesta a las necesidades de la sociedad en materia de vivienda. Este trabajo finaliza con el desarrollo de una serie de reflexiones orientadas a analizar cómo los crecientes fenómenos migratorios, ligados a asentamientos precarios, no tan solo plantean el desafío de la pobreza y la vulnerabilidad como tal, sino también de qué manera las exclusiones sociales, invisibilizadas dentro de las mismas, se constituyen como importantes retos tanto para el Estado, en su limitada esfera de acción, y para las organizaciones de la sociedad civil (OSC), como la Fundación Quiero Mi Casa, en su rol activo por la superación de estas exclusiones. 


\section{Antecedentes}

\section{Aproximaciones al concepto de "exclusión social" y su relación con los conceptos de "pobreza" y/o "vulnerabilidad"}

Desde la sociología temprana, las definiciones de "exclusión social" han emergido desde las aportaciones teóricas de Marx, Engels, Tönnies o Durkheim, y han ido de la mano del fenómeno de la alienación y del concepto de "clase social" (Jiménez, 2008). Es en la década de los 60 que un teórico francés como Rene Lenoir instala el debate teórico acerca de la exclusión formulando referencias vagas e ideológicas respecto de la figura de los pobres, hablando de ellos como excluidos (Lenoir, 1974). Posteriormente, el concepto fue aplicándose paulatinamente a diferentes categorías de desventaja social, incluyendo nuevos grupos y, por ende, diversos fenómenos sociales, con connotaciones y usos difusos.

Su posterior desarrollo desde los 70 y los 80 se enmarca directamente en el contexto social global, pero por sobre todo europeo.

Las consecuencias de los procesos de precarización postindustrial traerían a Francia el término "nuevos pobres", pues la exclusión no solo significó la identificación de aquellos individuos (los excluidos), incapaces de contentarse de los frutos atrayentes del progreso, sino también la afectación de las clases medias, históricamente beneficiadas con el pleno empleo, los contratos indefinidos y la salvaguardia del Estado Bienestar (Bachiller, 2013).

De esta forma, los teóricos franceses de dicha época caracterizaron la exclusión social de acuerdo con dos focos complementarios entre sí. Por un lado, la integración social del individuo gracias a su producción y, por otro, un elemento que involucra principalmente variables individuales o personales que dependen de la inclusión en redes familiares y de sociabilidad, es decir, vinculaciones primarias. Bachiller señala que, de cierta manera, existe una lógica de causalidad entre estas dos variables, en las que el desempleo, la precariedad laboral o la marginación económica pudie- 
sen desencadenar problemas en las vinculaciones primarias $y$, por tanto, imposibiliten la inserción social (Bachiller, 2013).

Como se mencionó, es evidente la impronta de la escuela sociológica de Emile Durkheim al momento de hablar de exclusión social, pues el trasfondo, según estos autores, está en la relación tensa entre sociedad e individuo, y la amenaza latente del quiebre de la cohesión social. El concepto de "exclusión" va más allá de una perspectiva económica, porque se centra en la desunión del tejido social, lo que genera oscilaciones dentro de estos vínculos sociales. Desde esta perspectiva, la exclusión social puede entenderse como pobreza y precariedad, más aislamiento social o pérdida de las vinculaciones primarias.

Así, son varios los autores que han convenido usar el concepto de "exclusión social" en su carácter multidimensional, utilizándolo en diversas teorías y para explicar múltiples fenómenos sociales, haciéndolo elástico e incluso contradictorio. De acuerdo con el exhaustivo análisis de autores como Leyton y Muñoz (2016), esta característica multidimensional y multipropósito de la exclusión social es una de las que genera mayor acuerdo entre distintos autores. En otras palabras, existe consenso teórico respecto de la amplia gama de usos que este concepto ha tenido desde sus primeros esbozos. Por otra parte, señalan ellos que estas dimensiones son "subrayadas especialmente para distinguir el concepto de exclusión social de la idea de pobreza tradicional, es decir, la pobreza entendida como la falta de recursos para satisfacer las necesidades de los individuos" (Leyton y Muñoz, 2016, p. 46). En este sentido, en contraposición con la pobreza, la exclusión social es teorizada como un proceso multidimensional, que consiste en la presencia u ocurrencia de diversos factores sociales, internos o externos, que afectan la participación de las personas en la vida económica, social, cultural y política (Levitas, Pantazis, Fhamy, Gordon, Lloyd y Patsios, 2007), recalcando además que estas dimensiones pueden acumularse o ser interdependientes entre sí. Por otra parte, y en concordancia con lo anterior, existen autores que señalan que la exclusión social 
es más un proceso que un estado (Gordon, Adelman, Levitas y Pantazis, 2000; Atkinson y Da Voudi, 2000).

En concordancia con lo anterior, la noción de "exclusión" presentaría algunos atributos ventajas por sobre la idea o concepción tradicional de "pobreza", dado que el primero sopesa un análisis principalmente dinámico de este fenómeno, mientras que el segundo se enfoca en un análisis estático que, hasta la emergencia de la multidimensionalidad en su uso en el último tiempo, se reflejaba en sus sistemas de medición, tanto a nivel académico como en el de las políticas públicas. En este sentido, los autores afirman que se desprende una crítica sobre el concepto de "pobreza" ya que, si bien este enfoque provee de cifras y ayuda a caracterizar individuos o grupos que se encuentran en un lado desventajoso, no tiene la cualidad de reconocer los elementos desencadenantes de dicha situación, así como tampoco las diversas consecuencias, efectos e impactos en la vida de estos con relación a las desventajas sociales que manifiestan. Mientras que el valor del concepto de "exclusión social" radica en su contribución a mejorar el conocimiento, es decir, posibilita una mejor comprensión de los cambios en la situación de las personas en el tiempo.

Si bien el concepto de "exclusión social" en la actualidad abarca una serie de actores, procesos y fenómenos, es posible destacar de él otras diferencias con el concepto tradicional de "pobreza", pues, en dicha línea, no solo considera los recursos económicos de los individuos -o de ellos en el ámbito del hogar-, sino que, además, en algunos de sus usos, pone el foco en los recursos de las comunidades locales. Autores como Graham Room (2000) señalan que el análisis bajo el alero de la exclusión social posibilita una identificación más directa de actores -individuos, hogares o comunidades- que se encuentren sufriendo múltiples desventajas o vulnerabilidades sociales, así como el porcentaje de degradación de sus recursos colectivos y de sus lazos con la sociedad, es decir, la no inclusión en ésta.

De esta manera, la exclusión social contribuye a la identificación de estas situaciones o niveles de no integración y vulnerabilidad 
social, de una forma más enmarañada que la que permite el sentido de pobreza tradicional.

La gradualidad de la exclusión social o la exclusión entre fenómenos o entre niveles es otra de las potencialidades que los autores citados reconocen del concepto, pues se identifican diversos grados o niveles (desventajas heterogéneas) en los que un individuo puede estar excluido, al mismo tiempo que es posible estar incluido o excluido de diversos procesos o dominios simultáneamente. Dicho de otro modo, nadie está totalmente excluido socialmente; puede estarlo en diferentes niveles o grados, al mismo tiempo que se puede sufrir exclusión social en diversos ámbitos o componentes de la vida social. Además de lo anterior, Room (1995) sostiene que la exclusión social puede fluctuar a través de la vida del individuo o del grupo, y manifestarse en mayor medida durante algunos instantes claves, por ejemplo, en el nacimiento, deceso o en cambios laborales.

Considerando lo anterior, las nociones de "exclusión social" y de "pobreza" han de ser entendidas como términos que son necesariamente complementarios. Esto cobra particular relevancia para el análisis de la exclusión social en entornos latinoamericanos y, también, en el de Chile actual, dentro de los cuales hay una importante intercalación entre estas dos ideas (Leyton y Muñoz, 2016). De lo anterior se desprende que el concepto de "exclusión social", más que una alternativa al concepto tradicional de "pobreza", es una de las formas de abordarlo. Por ende, "este foco específico es provisto por el carácter relacional de la exclusión social. En este sentido, la exclusión social es comprendida como una forma de hacer explícitas las relaciones de poder que subyacen a la pobreza y la desigualdad" (Leyton y Muñoz, 2016, p. 48).

\section{Inclusión social versus exclusión social en asentamientos precarios}

Cuando hablamos de inclusión social en asentamientos precarios, gran parte de los significados que este concepto conlleva es- 
tán relacionados con sucesiones vinculantes con la ciudadanía social, es decir, con los derechos y libertades elementales de las personas que residen en asentamientos y que se relacionan con su bienestar (trabajo, salud, educación, formación, vivienda y habitabilidad, calidad de vida). De esta forma, según el Banco Mundial, "la inclusión social es el proceso de empoderamiento de personas y grupos, en particular los pobres y los marginados, para que participen en la sociedad y aprovechen las oportunidades" (OEA, 2011, p. 38). La comunidad, entonces, se constituye en una oportunidad para la inclusión, siempre y cuando influya en las políticas hacia las personas. Desde esta perspectiva, el término "inclusión social" también puede ser entendido como "producto de la agregación a determinados momentos de la sociedad. La agregación se producirá a través de unidades menores de la comunidad, la familia, y la residencia" (Azuero, 2009, p. 159). De acuerdo con lo anterior, el acompañamiento es esencial en estos tipos de variabilidad, pero, al mismo tiempo, es necesario que el interés por entender a la persona en su interacción con el ambiente incluya el análisis de los diversos materiales del desarrollo humano. De esta forma, el rol que cumplen los actores e instituciones sociales influye en la construcción de sociedades inclusivas.

Así, se deduce que es contingente caminar hacia la inclusión social en asentamientos precarios, en la medida en que se aborden factores asociados a la exclusión y, desde estos, proyectar propuestas que busquen su solución, entendiendo que esta exclusión es opuesta a la inclusión social y necesariamente relacionadas entre sí. La exclusión social en asentamientos precarios involucra una imagen dualista de la sociedad, en la que suelen convivir dos sectores: uno "incluido" y otro "excluido".

Desde el punto de vista sociológico, sin embargo, se plantea que los conceptos de "inclusión” y "exclusión" social "han sustituido en nuestros discursos a los oprimidos, a los dominados y a los explotados de otros tiempos. No es casual: si hay oprimidos, hay opresores, si hay dominados, hay dominadores y si hay explotados, hay explotadores" (Rodríguez, 2012, p. 34). En cambio, según 
Sawaia (2004), suele entenderse como un dualismo, que percibe aquellas nociones de los "excluidos" y los "incluidos" de formas más bien paralelas, debido a cierta oposición entre ellas, lo que desencadena una relación dialéctica y reproducción de las relaciones de poder por parte de la idea de "exclusión".

\section{Exclusiones observadas dentro de los asentamientos precarios: migración y racialización}

Cuando se habla de fenómenos dentro de los asentamientos precarios se puede encontrar en estos diversas exclusiones, entre las cuales se observa la marginalidad, que provendría en este caso de los habitantes de aquel espacio circundante, entendiendo también este contexto como un espacio invisibilizado y por tanto segregado por la sociedad. La marginación da cuenta entonces de entendimientos sobre la base de diversas formas; Bunges (2008) comprende este concepto como la exclusión, por parte de los individuos, de al menos uno de los tres elementos del subsistema social, es decir, dentro de los parámetros de lo económico, político y cultural. Al mismo tiempo, destaca que mientras más marginal sea el individuo, "tanto menos obligado se siente a obedecer los códigos moral y legal vigentes en la sociedad" (Bunges, 2008, p. 67).

Teniendo como apoyo la definición de marginalización, se puede profundizar en las diferentes problemáticas, las que estarán ligadas a temas convergentes, como la migración y racialización.

De esta forma, el fenómeno de la migración en Latinoamérica, según Carolina Stefoni (2005), se ha ido dando de acuerdo a tres patrones: la migración de ultramar, situada en los siglos XIX y $\mathrm{XX}$, que tiene como principal característica la llegada de europeos al territorio sudamericano, dando cuenta también del arribo del desarrollo y la modernización; la migración intrarregional, determinada principalmente por temas económicos y políticos de los países, y la migración hacia Estados Unidos por parte de personas con nacionalidades del continente sudamericano. Lo que ocurre en Chile es la migración interregional, la cual, actualmente, de 
acuerdo con el INE, es cuatro veces mayor que lo que acontecía al final de la dictadura cívico-militar (INE, 2018).

Siguiendo con Stefoni (2005), los inmigrantes provenientes de países vecinos aumentaron en la década de los noventa, en paralelo con la disminución de inmigrantes originarios de Europa. Esto, sin embargo, generó consecuencias dentro del imaginario social, dado que "en el imaginario colectivo quedó la idea del inmigrante blanco, masculino, europeo y poseedor del conocimiento" (Stefoni, 2005, p. 3). Este ideal de estereotipo extranjero por parte de la sociedad chilena generó una oposición contra los nuevos inmigrantes, lo que trajo como consecuencia una continua marginación de los extranjeros procedentes de países andinos.

En otras palabras, la inmigración, al ocurrir con personas provenientes de países aledaños, dio pie a que hubiese esta diferenciación entre inmigrantes europeos y sudamericanos u otras nacionalidades, que no traen consigo necesariamente la simbolización propiamente tal de modernismo y desarrollo (Stefoni, 2005).

Asimismo, la idea de la "racialización" se entiende como algo que, en lo preponderante, convoca a que un individuo cumpla con ciertas características, exclusión que se hace más visible mediante la conceptualización de un "otros". La racialización puede entenderse mediante la siguiente definición: "no existen grupos raciales per se, sino solamente grupos socialmente racializados como resultado de prácticas, doctrinas y voluntariosas producciones de saberes" (Campos, 2012, p. 2). Esto genera cierta división de la misma comunidad, categorizando lo que se considera como lo homogeneizante y común, que además tiende a la producción de la heterogeneización de ciertos grupos.

Esta terminología, según Campos (2012), suele ser utilizada en lo concerniente a las políticas públicas, al igual que dentro de las demandas de movilizaciones sociales, intentando dar cuenta finalmente de la holgada desigualdad e inequidad entre los diversos grupos sociales adscritos a una misma estructura societal. 
Además, aquella categorización y, por tanto, las divergencias entre uno y otro grupo son insustanciales si es que alguno de ellos carece de existencia, vale decir, sin la noción de lo "no blanco" no se categorizaría per se lo "blanco", ni se distanciarían uno de otro por sus características. Es decir, aquellas disociaciones son reproducidas por el simple hecho de que estas diferencias son culminadas y perpetuadas en el tiempo; sin embargo, la racialización no es necesariamente universal, sino que, más bien, son los territorios quienes se apropian de ella y la van resignificando.

De esta forma, los diversos fenómenos de exclusión social, tanto la migración como la racialización, generalmente son abordados desde una intervención que radica en cómo contribuir a la superación de estas mismas desde diversos enfoques del trabajo social y las políticas públicas.

\section{Intervención social en asentamientos precarios}

Desde el Trabajo Social, el tratamiento de la inclusión y la exclusión social en asentamientos precarios permite hacer hincapié en la subjetividad de la persona involucrada. Su examen se apoya en una consideración tanto política como ética: "La primera, se expresa en la intencionalidad de transformación social y de alcanzar mayores niveles de justicia social y la segunda supone la preocupación por el sufrimiento humano y el compromiso con su superación" (Rodríguez, Pierri, Ana, Da Silva, Rossi y Castro, 2013, p. 96).

Asimismo, es necesario tomar en cuenta los nuevos fenómenos sociales intervinientes en los asentamientos precarios que generan exclusión social, tales como los flujos migratorios, en los que la intervención social desde el Trabajo Social aporta antecedentes que orientan a revisar sus objetivos y estrategias cuando convoca la participación de migrantes en la comunidad desde una mirada más amplia, que acoge y responde a la diversidad, en este caso racial, lingüística e idiosincrático-cultural. En este mismo sentido, el acompañamiento psicosocial efectivo en los asentamientos tam- 
bién podría develar mecanismos facilitadores u obstaculizadores que operan en la inserción social de los migrantes, lo que contribuiría a orientar y enriquecer los avances en políticas públicas y sociales que hoy se llevan adelante en estas materias.

Para contribuir a la superación de estas exclusiones, si bien destaca la labor de la intervención social desde el trabajo social y la sociedad civil, se entiende como un deber que el Estado sea garante en mayor medida para dicha contribución. Por esto, es necesario recorrer las diferentes nociones de la política pública, y en ella la política social, para posteriormente mencionar su trayectoria en materia de vivienda en Chile.

\section{Políticas públicas y exclusión: abordaje desde las políticas diseñadas e implementadas por el Estado}

Muchos autores concuerdan definir "políticas públicas" como las acciones (o inacciones) que ejerce el Estado para dar solución a ciertos problemas de carácter público (Olavarría, 2007). En lo concreto, se entiende "políticas públicas" como aquellas decisiones tomadas desde las esferas públicas y materializadas en instrumentos de gestión para responder problemas definidos como públicos por los actores de poder. Ahora bien, más allá de ser entendidas como respuestas técnicas que pudiese dar el Estado para hacer frente a las necesidades de la población, las políticas públicas, y en específico las políticas sociales, buscan configurarse como el principio de igualdad, integración e inclusión social que orienta su accionar hacia un proyecto de sociedad cohesionada.

Respecto a la asistencia y protección social, para Castel estas políticas "están dirigidas a otorgar protección social al mundo obrero en un contexto de acelerados procesos de industrialización, urbanización y expansión del modo de vida urbano. La protección social asume la forma de derechos laborales" (Castel, 1997, p. 232). Con el pasar de los años, apreciamos que este enfoque se dirige hacia otorgar beneficios sociales a la población en extrema pobreza, teniendo como objeto su inserción en la sociedad. 
En Chile, la trayectoria de las políticas de protección social se ve transformada por las reformas realizadas en 1980, al introducirse lógicas de mercado en áreas como educación, salud, vivienda y seguridad social, pasando del Estado de Bienestar a uno de tipo residual, que puso un mayor énfasis tanto en la acción pública como en la población más pobre y carente, y depositó en los individuos el compromiso del logro de su bienestar (Esping, 1990). La presencia de una nueva pobreza urbana, favorecida en un primer momento por el fenómeno migración campo-ciudad de inicios de los años 50 y su ocupación territorial en las denominadas "poblaciones callampas", en donde, para 1952, vivían unas 75.000 familias, las tomas de terreno de los años 60 y la posterior traducción de estos asentamientos precarios en los denominados "campamentos", comienzan a desafiar de manera potente el rol del Estado en materia habitacional (De Ramón, 2006).

El fenómeno de la precariedad urbana, entendida como la "proporción de hogares que no tienen cubiertas sus necesidades habitacionales, tales como materialidad de la vivienda, acceso a servicios (agua y saneamiento) y la tenencia" (Winchester, 2008, p. 28), se configuraba como uno de los principales problemas a los que debían responder las políticas públicas. A partir de la dictadura militar (1973-1990), el Estado toma una función subsidiaria, las familias en extrema pobreza son vistas bajo un enfoque asistencialista, es decir, como receptores de beneficios para la adquisición de viviendas según la oferta presente en el mercado privado. En este periodo, el Estado "abandona labores de financiamiento y construcción de viviendas permanentes, para concentrarse en el subsidio habitacional y las viviendas sociales" (MINVU, 2007, p. 184). Pese a los intentos del Estado (relegando gran parte de sus funciones al mercado) por llevar a cabo una política habitacional que diera respuesta a las fuertes demandas de la población en extrema pobreza, para mediados de los años 80 comienza el resurgimiento de nuevos campamentos. El Catastro Nacional de Marginalidad 46 Habitacional, realizado por el Ministerio de Vivienda y Urbanismo (MINVU) en 1984, registra exactamente 482 
asentamientos del tipo campamento y 40.493 familias habitando en ellos (MINVU, 1985).

Siguiendo con los datos del MINVU (1985), en 1990 el déficit acumulado alcanzaba las 949.295 viviendas y el 52\% correspondía a viviendas que requerían ser reemplazadas, como lo eran las viviendas asociadas a asentamientos precarios (viviendas irrecuperables, según la nomenclatura del déficit habitacional). Durante esta etapa, se hizo hincapié en la construcción masiva de viviendas bajo el enfoque subsidiario y focalizado en el periodo anterior, "construyéndose anualmente más de 96.000 soluciones habitacionales con alguna participación del Estado" (MINVU, 2007, p. 231). Entre 1990 y 1996, la deuda habitacional se redujo en un $42 \%$ y las viviendas precarias pasaron a ser el 37\% del total (Encuesta de Caracterización Socioeconómica [CASEN] 1990, 1996). No obstante, la masividad tuvo nefastas consecuencias en "el crecimiento desequilibrado de las ciudades y el surgimiento de zonas periféricas homogéneas, en la condición socioeconómica desmejorada de sus habitantes y con insuficiente dotación de servicios urbanos" (MINVU, 2009, p. 12).

No obstante, a inicios del 2000 "aún existía un núcleo de pobreza extrema que era refractaria al impacto de las políticas sociales, por lo que se diagnosticó, como una de las razones principales, que esos grupos no accedían a la oferta pública" (Larrañaga, 2010, p. 10). Ese núcleo, colmado de pobreza, se calculó en $225 \mathrm{mil}$ familias que representaban un 5,6\% de la población nacional, y que careció de los beneficios asociados a las políticas implementadas, dado que estas obtuvieron un escaso efecto (Palma y Urzúa, 2005).

En respuesta a lo anterior, surgen nuevos programas focalizados en la atención a los campamentos. Según lo expone Rivas (2013), un ejemplo de ello es el Programa Chile Barrio, enmarcado en el Plan Nacional de Superación de la Pobreza. La habilitación social dentro del Programa era la búsqueda del fortalecimiento de la organización y, por correspondencia, de la participación comunitaria. Aquella se estructura alrededor de dos componentes: el Ser- 
vicio de Desarrollo del Barrio (SDB) y Un Barrio para mi Familia (UBPF). Sin embargo,

según resultados del Catastro 2011, se muestra un alza en el número de asentamientos y familias habitando en ellos con respecto a la última medición. Este aumento corresponde a 167 nuevas unidades territoriales que responden a las características de un campamento ( $34 \%$ de variación) y coincide con un periodo sin una línea de trabajo particular para campamentos, lo que demuestra que este tipo de asentamientos no fue absorbido por la línea regular como se esperaba. (Rivas, 2013, p. 52)

Lo anterior deja entrevisto que las políticas habitacionales enfocadas en la entrega de subsidios no han sido suficientemente sustentables para erradicar los asentamientos precarios, pues, tal como lo reflejan las cifras, muchas de las personas que han salido de los campamentos vuelven a ellos, sigue entrando nuevas personas y se forman nuevos reductos.

Las líneas generales de acción de las políticas públicas en materia de vivienda tienen un eje en común, y es su tendencia a la centralización, desde la cual se acota su accionar al solo hecho de la entrega de beneficios, sin generar instancias efectivas de participación de la comunidad. Las generadas solo resultan ser consultivas y no vinculantes para un proceso de toma de decisiones, lo que solo ha permitido otorgar respuestas y soluciones parciales y estándar a las necesidades de la población que aún reside en asentamiento precarios, sin considerar que entre ellos existe una notable diferencia, por cuanto sus lógicas, características de las familias que los componen, dinámicas, difieren de manera importante las unos de las otras.

Por último, los países cuentan con diferentes mecanismos para abordar la inclusión social. Por lo general, tienen el carácter multidimensional. Así, en el área de vivienda, la inclusión social hace referencia a que todas las personas tengan acceso a una vivienda habitable, es decir "cómoda, suficiente y confortable" (MINVU, 2018, p. 1). Sin embargo, la política habitacional actual no da cuenta necesariamente de este principio. Según dichos de la pro- 
pia ministra de Vivienda y Urbanismo en 2014: "el déficit cualitativo de viviendas supera al déficit cuantitativo, por lo que se hace necesario fortalecer los programas de mejoramiento de viviendas existentes que, a su vez, se enlazan con otros planes y programas urbanos" (MINVU, 2014, p. 1). Esta declaración sugiere que, al menos para el caso de las políticas habitacionales chilenas, aún no se cuenta con una distribución que permita la inclusión de los sectores más carenciados socialmente, tales como las personas residentes en asentamientos precarios.

Se tiene entonces que la ley chilena, en sus variados ámbitos de intervención, ha estado dirigida a grupos vulnerables. Sin embargo, su desarrollo ha estado limitado a la focalización y a factores económicos que inciden en el financiamiento de viviendas, lo cual no permite avanzar de manera efectiva en una real política social inclusiva. El factor de desigualdad, ligado a la mala distribución de oportunidades y de ingreso, junto a una falencia de una estrategia de desarrollo social, no permitirían un logro adecuado de estos indicadores mencionados, por lo que se entiende que es un trabajo por realizar.

Así, sobre la base de los antecedentes expuestos, se desprenden para el presente artículo, los siguientes objetivos:

\section{Objetivo general}

Conocer cómo ciertos fenómenos de exclusión social, detectados en asentamientos precarios en Chile, desafían a las políticas públicas y a las organizaciones de la sociedad civil (OSC) que buscan superarlos.

\section{Objetivos específicos}

- Describir ciertos fenómenos de exclusión social presentes en los asentamientos precarios.

- Conocer los desafíos de estos fenómenos de exclusión para las políticas públicas del Estado chileno.

- Conocer los desafíos de estos fenómenos de exclusión para las OSC. 


\section{Metodología}

Respecto de los aspectos metodológicos del presente artículo, estos refieren a un estudio de caso y también a la revisión bibliográfica y de experiencias concernientes a los desafíos de la exclusión social para las políticas públicas y las organizaciones de la sociedad civil, tanto en un nivel general como en aquellas expresiones que se manifiestan en fenómenos como la migración, que aquí denominaremos como "subexclusión".

Por lo tanto, fue necesario el ordenamiento de información de algunos datos secundarios, entendidos estos como la información que permite conocer hechos o fenómenos a partir de documentos o datos recopilados por otros (Guzmán, 1982), provenientes de instrumentos y mediciones nacionales, como la Encuesta CASEN, el Registro Social de Hogares, datos del Catastro de Campamentos del Ministerio de Vivienda y Urbanismo, del Instituto Nacional de Estadísticas (INE), o encuestas que aluden a cifras sobre migración, las cuales, precisamente, aportan datos concretos sobre estas subexclusiones a las que se hace referencia a lo largo de todo el análisis.

Además de recurrir a la bibliografía y al marco de antecedentes que describe y detalla el planteamiento de las exclusiones sociales, se hace referencia aquí a un estudio de caso, concerniente a la experiencia y al trabajo de Fundación Quiero mi Casa como organización de la sociedad civil que interviene en contextos de vulnerabilidad y exclusión social, en campamentos con población migrante en donde, de cierta manera, dichas exclusiones se hacen presentes. Se entiende por "estudio de caso" aquel esquema comprensivo en el cual están reunidos y ordenados todos aquellos datos relevantes y significativos correspondientes a una experiencia concreta de un individuo o un grupo de éstos (Ander-Egg, 2017). Para el autor, este método permite al investigador "ordenar la información acerca de una persona, con el objetivo de realizar un diagnóstico que posibilite plantear un proceso de cambio o la modificación de una situación” (Ander-Egg, 2017, p. 30). Como se 
mencionó, el caso al que se alude forma parte del trabajo realizado por Fundación Quiero mi Casa, ante los desafíos para inclusión social desde una arista tan relevante como la migración.

Volviendo a los datos secundarios, Scribano y De Sena (2009) señalan que la utilización de fuentes secundarias posee dos variantes en del marco de una investigación social. Por una parte, el uso de esta información permite al investigador revisar la bibliografía en un nivel específico sobre el tema que se trabaja, además de permitir establecer qué se ha escrito y planteado ya sobre éste, si existen otras perspectivas de estudio y cuáles son, la existencia de los principales posicionamientos sobre la materia, cuáles han sido los principales hallazgos hasta ahora, entre otros elementos; es decir, una suerte de "estado del arte" de dichos fenómenos, para utilizarlos como un marco de antecedentes o un marco teórico.

Por otra parte, se puede utilizar estos datos como material empírico, es decir, como un objeto de estudio en el que el uso de dicha información permite contrastar los planteamientos investigativos propuestos al inicio del análisis, suponiendo así una fuente de información de primer orden para la investigación sobre la que se pueda trabajar (Scribano y De Sena, 2009).

Del mismo modo, la labor de la revisión bibliográfica constituye una etapa esencial para todo proyecto de investigación, reflexión o análisis, debiendo garantizar que se obtenga la información más relevante en el campo de estudio determinado, en este caso, desde las ciencias sociales, a partir de un amplio universo de documentos (Gómez, Fernando, Aponte y Betancourt, 2014).

A partir de lo anterior, se debe mencionar que, al igual que respecto del uso de material secundario, se realiza una revisión bibliográfica de los conceptos orientadores del análisis, a saber, la exclusión social, la intervención social, las políticas públicas, entre otros, al mismo tiempo que se revisa la literatura acorde a los fenómenos y/o problemáticas adyacentes a estos conceptos, como lo son la migración, la racialización, la diversidad sexual y el género, vale decir las subexclusiones ya mencionadas. En este sentido, los 
autores definen la revisión bibliográfica o estado del arte como "la descripción detallada de cierto tema o tecnología, pero no incluye la identificación de tendencias que puedan plantear diferentes escenarios sobre el desarrollo de la tecnología en cuestión y que permitan tomar decisiones estratégicas" (Gómez, et al., 2014, p. 158).

Según estos autores, la metodología correspondiente a la revisión bibliográfica puede aplicarse a cualquier tema de investigación para establecer la importancia de este, asegurando la originalidad del estudio. Al mismo tiempo, permite que otros investigadores consulten las fuentes bibliográficas utilizadas, pudiendo comprender lo investigado y quizá continuar o proyectar a otras temáticas el trabajo realizado (Gómez, et al., 2014).

\section{Exclusión social y desafíos para su superación}

cuando se observa el fenómeno de la migración en los asentamientos precarios, se determina un proceso de adaptación e integración de los migrantes a los espacios urbanos, lo cual lleva a prácticas discriminatorias por parte del nacional hacia el migrante, generando segregación de estos grupos minoritarios.

De acuerdo con Iturra (2016), es posible observar que, en Santiago, prácticas como la intolerancia étnica, racial y cultural no son observables directamente por medio de actos de violencia explícita, sino que estas se manifiestan de manera tácita en hechos como la desigualdad salarial, precariedad laboral o actitudes discriminadoras asociadas a la tenencia o arriendo de viviendas. De acuerdo con lo anterior, la reacción propia de los inmigrantes se manifiesta en la reunión en un mismo espacio territorial y el refuerzo de la identidad propia, lo cual, según Marcuse (2001) pudiese reconocerse como "enclaves étnicos".

De esta forma, tal como lo señalan Hidalgo y Torres, "estos inmigrantes habitan la ciudad y se apropian del espacio, llevando a él toda su historia personal y expectativas de vida" (Hidalgo y Torres, 2009, p. 310). La cultura, conocimientos y prácticas se hacen presentes en estos espacios comunes del asentamiento precario, 
sobrepasando el objetivo principal con que el inmigrante llega al lugar, el cual no solo es habitar, sino que, además, relacionarse con los demás pobladores.

En este proceso de integración se va generando una identidad colectiva, reforzando prácticas comunes, pero adquiriendo al mismo tiempo nuevas costumbres, produciéndose así un proceso de aculturación. En la práctica, para el caso de los asentamientos precarios, este proceso resulta mucho más evidente, pues quien llega es recibido por sus pares de manera más efectiva, ya que existe una noción de intencionalidad comunitaria. Siguiendo con Iturra,

la apropiación o bien la ocupación de un espacio público, tiene que ver con practicar en éste, dinámicas cotidianas propias del lugar de origen, combinándolo con la cotidianidad local, entrelazándose en un sincretismo de identidades tanto individuales como colectivas, incorporando elementos propios del migrante en el territorio de la sociedad de destino, resignificando los espacios públicos y privados a partir de sus usos, gustos y costumbres. (Iturra, 2016, p. 28)

Por otra parte, cuando se habla de territorio se circunscribe la definición de asentamientos precarios como la "falta de servicios básicos, vivienda por debajo de los estándares o ilegal, y estructuras inadecuadas de construcción, hacinamiento y alta densidad, condiciones de vida insalubres y localización en zonas de riesgo, inseguridad en la tenencia, asentamientos informales o irregulares, pobreza y exclusión social" (UN Habitat, 2010, p. 68). Estos asentamientos con alta presencia de inmigrantes se constituyen como espacios de protección del grupo frente a otros grupos étnicos, en los que, como se mencionó, se realizan una serie de prácticas culturales, otorgándoles un sentido de pertenencia social y étnica. Resulta evidente que el arribo de inmigrantes de bajos ingresos pudiese propiciar una informalidad en el habitar, como por ejemplo la llegada a asentamientos precarios, tales como campamentos o conventillos. Es tácita entonces la dificultad de la población inmigrante para acceder a la vivienda definitiva, pues se enfrenta 
a dificultades sobre todo en la Región Metropolitana, región que, por cierto, concentra el mayor número de este colectivo.

En este sentido, las políticas habitacionales en Chile, hasta 2015, solicitaban un Certificado de Permanencia Definitiva (CPD) con una antigüedad superior a 5 años, que, sumados a otros 5 anteriores de residencia en el país, previos a la obtención de este documento, dificultaba aún más el acceder a un proyecto habitacional. Sin embargo, en la actualidad esto ya no ocurre, pues dicha causalidad ha sido eliminada, pero aun así existen tareas pendientes respecto de la política migratoria, ya que, por lo que se ha podido observar - por ejemplo en las intervenciones sociales en las cuales trabaja Fundación Quiero Mi Casa, ONG chilena dedicada a la intervención social en asentamientos precarios en la Región Metropolitana-, el enfoque de trabajo debe ser inclusivo y no integrativo, y debe haber coordinación de redes entre el sistema público, el sistema privado y la sociedad civil.

De la misma manera, en los asentamientos precarios en los que trabaja dicha ONG se observa que las intervenciones desde el Estado se ciñen a los modelos de intervención preestablecidos por los mismos programas y proyectos que generalmente carecen de un enfoque intercultural, y prevalece el enfoque de integración social, como el plan de atención a migrantes "Chile te Recibe", plan piloto ejecutado por el gobierno de Chile el año 2017, que facilitaría el acceso a información a las persona migrantes que residen en el país. De esta forma, las intervenciones sociales en asentamientos precarios no tan solo plantean, tanto para el Estado como para las ONG, el desafío de la pobreza y la vulnerabilidad como tal, sino que también las exclusiones sociales invisibilizadas dentro de las mismas, tales como la migración.

Asimismo, cuando se interconecta la migración con la exclusión social dentro de estos espacios precarios, es importante mencionar que existe divergencia en el trato a los migrantes provenientes de Europa y los que provienen de países vecinos, dado que la sociedad chilena tiene en el imaginario colectivo al inmigrante eu- 
ropeo, que aportaría a la sociedad in situ modernidad y desarrollo, mientras que al migrante de países aledaños se le otorga un sitial que no es de privilegio, sino que, por el contrario, tiende a ser discriminatorio.

Esto alerta a que, ya desde el imaginario, se va construyendo una suerte de discriminación y, por lo tanto, se sucumbe hacia una fuerte exclusión social de quienes son partes de este colectivo y poseen ciertas características, categorizándolos en aquellos grupos subordinados. De acuerdo con Baeza (2008), aquello propende en una diferenciación en torno a dos conceptos claves; el primero tiene relación con el concepto de "xenofilia", que se entrelaza con el de migrante y que cumple con un perfil europeo; en cambio, para quienes se categorizan de acuerdo con un estereotipo correspondiente al migrante andino se vuelve imperativo el término "xenofobia". Lo anterior lleva a deducir que, a nivel macro, existe también una relegación y, por lo tanto, una marginación de aquel estereotipo de inmigrantes.

Volviendo entonces a los datos de la CASEN (2015), esta corrobora que el 23\% de aquellas personas sufre de pobreza multidimensional y que se ratifican con mayor claridad en los campamentos emplazados en la región de Atacama, los cuales tienen la característica de que sus miembros proceden en su mayoría de países como Bolivia y Perú. Es importante destacar también que el total de la población nacional que se encontraba en aquella situación de precariedad en 2015 bordeaba el 21\% (Campos, 2017).

Entonces, si se entiende aquello como una subordinación existente dentro del constructo social, también debería coexistir y vivenciarse en aquellos sitios que están segregados socialmente. En este caso, dentro de los asentamientos también correspondería entrever aquella discriminación como una suerte de doble exclusión, dado que la categoría de inmigrante originario de un país no es bien vista socialmente, comprendiendo que, dentro de grupos más cerrados se suelen reproducir estos patrones culturales emergentes; "en todas las capas sociales y económicas aprendemos a 
'despreciar' al otro/a, al que no se enmarca en el modelo de lo blanco, patriarcal, adulto, heterosexual, sano y pudiente" (Benalcázar, 2001, p. 7).

Por este motivo, el rol del trabajador social y la intervención que éste realiza en los asentamientos precarios es relevante, ya que permite reducir ciertas trabas que coartan una participación e inclusión social efectiva de los migrantes. En este sentido y de acuerdo con autores como Miguel y Zarelli Fonseca (2004), desde el Trabajo Social, lo relacionado con el reconocimiento de las diferencias y la aceptación del otro como legítimo otro son los ejes centrales en los procesos de comprensión de lo social. Desde los marcos de comprensión de la disciplina se reconoce que

la apelación a lo diverso, a una apertura a lo diferente, a la promoción de cambios culturales, no pocas veces es recibida con temor. Entre otras razones, esto se caracteriza por un recelo a la idea del otro. Esto ha sido fuente de un riquísimo imaginario sociocultural, poblado de visiones amigables o amenazantes. Desde la idea de otro natural intrínsecamente bueno y que poco a poco se pervierte en el contacto social, a la idea de bárbaros. Toda la noción de civilización y barbarie radica en la distinción de la idea de otro. (Matus, 1999, p. 87)

En concordancia con lo anterior, se debe entender que, actualmente, la disciplina del Trabajo Social analiza y busca hacerse cargo de las rupturas de las nociones de identidad tradicional. Es así como los autores afirman que es posible una mirada compleja para comprender estos fenómenos, la cual trasciende lo dual, es decir, aquellos esquemas de comprensión tradicionales (Fonseca y Fonseca, 2004).

Por lo anterior, innovar en estrategias de intervención, incorporando la mirada de las personas, es fundamental al momento de trabajar con migrantes en asentamientos precarios, pues ahí radica el cambio de enfoque, pasando desde lo integrativo a lo inclusivo. De esta forma podemos observar, por ejemplo, el modelo de intervención social de Fundación Quiero Mi Casa, el que destaca 
por su acompañamiento a las personas residentes en estos asentamientos, ya que su propuesta se basa en etapas, las cuales, desde determinado momento, permiten una intervención más flexible en cuanto a actividades, modelos de trabajo y metodología, estimados a ser trabajados durante tres años aproximadamente, incluyendo labores de diagnóstico, trabajo en terreno y habilitación social, además del monitoreo antes de su cierre. Estas etapas se guían a partir de componentes que sirven de hojas de ruta, que la intervención debe ir cumpliendo para que así el trabajo resulte exitoso.

El modelo incluye una etapa 0 de pre-intervención que posibilita la preselección de nuevos asentamientos, al mismo tiempo que incluye etapas que permiten la habilitación social tanto en organización comunitaria como en capacidades de ahorro y generación de ingresos. (Fundación Quiero Mi Casa, 2019, p. 3)

Desde Fundación Quiero Mi Casa esto se desarrolla desde un punto de vista promocional, en el que, para llevar a cabo la intervención, se trabaja con pobladores chilenos y migrantes de los asentamientos precarios, en niveles comunitario y/o familiar, en conjunto con estudiantes en práctica y/o voluntarios de Trabajo Social, destinando gran parte de los recursos a llevar a cabo iniciativas con sentido para los residentes del lugar. Al posicionarse en el territorio como Fundación y conocer a los residentes, establece un proceso metodológico inicial, para luego llevar a cabo una serie de actividades de índole social, en las que la comunidad es el centro de la intervención, creando conciencia en torno a diversas problemáticas que rodean el territorio, activando espacios, conociendo la historia del asentamiento, respetando y cuidando el territorio, apropiándose del asentamiento e identificando lo positivo y los recursos. Este trabajo se lleva a cabo incorporando a niños, adultos y adultos mayores, de manera que exista una integración intergeneracional.

De esta forma, la participación activa de los diferentes residentes es fundamental, ya que permite la integración, unificar criterios 
y dejar de lado elementos de subexclusión social, tales como la discriminación por ser migrante, y la comunidad se conforma como un ente propio, dando identidad y respeto común. Al mismo tiempo y al igual que muchas organizaciones de la sociedad civil que trabajan en las temáticas de pobreza, vulnerabilidad y, por tanto, en la superación de la exclusión social, uno de los principales desafíos se refiere a la mirada asistencialista de las políticas sociales, ya provengan desde el Estado, como de otras instituciones privadas sin fines de lucro que trabajen en la materia. De acuerdo con Codoceo y Muñoz (2017), la subsidiariedad de las instituciones trasforma la política social en un ejercicio asistencialista, puesto que se da por sentado que será el Estado y sus instituciones pertinentes quienes proveerán a la población que, de una u otra forma, ha quedado excluida del margen de acción y acceso a los servicios tradicionales: salud, educación, vivienda, trabajo, seguridad social, etc. Debido a esto, los autores sostienen que

las percepciones y valoraciones que se tienen sobre las políticas públicas están atravesadas también esencialmente por índices de beneficio personal. Dicho de otra manera, la implementación de las políticas públicas es evaluada positivamente si ellas, directa e indirectamente, favorecen al sujeto consultado. (Codoceo y Muñoz, 2017, p. 384)

Esta visión utilitaria de la institucionalidad desde quienes la reciben, adscribe al primer desafío que tanto las políticas públicas como las OSC han de superar, pues dificulta las metodologías de habilitación social o desarrollo de capacidades, además de generar expectativas que, debido a la condición de subsidiariedad, el Estado nacional no puede cubrir por completo, por ejemplo la de pobladores de asentamientos precarios.

Por otra parte, el resurgimiento de los asentamientos precarios ya no solo obedece a una problemática habitacional, sino que también a una multiplicidad de factores que dificultan la calidad de vida de la población que reside en estos lugares: el país de origen, el empleo al que se accede se muestran como fuertes limitantes 
que van a incidir de manera directa en el aumento de las brechas sociales existentes en Chile, y que van a potenciar el fenómeno de exclusión que viven estas personas.

Estos nuevos contextos se configuran como importantes desafíos a los cuales el Estado debe hacer frente. Sin embargo, los intentos por generar políticas que intenten abordar de manera integral no solo el déficit y calidad habitacional, sino también el hecho de fortalecer las capacidades y capital social de las personas que residen en estos asentamientos, y contribuir en su esencia a la cohesión social, han quedado en deuda, dejando en claro el rol poco activo del Estado por contribuir desde la política social en la garantía de derechos básicos para la sociedad.

En vista de los múltiples desafíos que supone la exclusión social en los asentamientos precarios, en términos de pobreza multidimensional, precariedad habitacional y acceso a oportunidades, como es poder optar a una solución habitacional que cumpla con los estándares de calidad, acorde al derecho a la vivienda adecuada (Observatori DESC, 2013), son múltiples también los actores públicos o privados, individuales o colectivos- que pueden hacerse cargo de la elaboración y ejecución de las diversas estrategias que vayan en pos de la superación de estas exclusiones.

Los desafíos del Estado, de acuerdo con las temáticas de migración, se expresan en primer lugar en relación con ciertas leyes que no amparan debidamente a los inmigrantes, puesto que, de acuerdo a Colomés y Pávez (2018), el decreto ley de extranjería promulgado dataría de $1975 \mathrm{y}$, desde entonces, solo se han promulgado las leyes $\mathrm{n}^{\circ} 20.430$ de refugiados y $\mathrm{n}^{\circ} 20.507$, acerca de tráfico y trata, aunque, si bien se han presentado dos proyectos de ley, en 2013 y 2017, aún se debaten en el parlamento.

Esto necesariamente genera una crítica, según los autores, la cual radica en la carencia de una política migratoria integral, dado que aquellas leyes ya promulgadas denotan una situación estratégica por parte del Estado en base a políticas aisladas, es decir, que "no 
responde[n] a los principios de derecho que la democracia exige" (Colomés y Pavez, 2018, p. 115).

Sintetizando lo anterior, los desafíos que posee el Estado frente a estas exclusiones deberían ir encauzado hacia la protección de los derechos de los inmigrantes. De esta forma, según lo expresa Colomés y Pavez (2018), se debe visualizar una necesidad de acuerdo con la adecuación de la legislación vigente, teniendo en cuenta la realidad migratoria que se vivencia en el país.

Por otro lado, la creación en 1990 de un sistema de indicadores para la medición del desarrollo humano y social, sumado a las mediciones mismas de la pobreza y, por ende, de la exclusión social en los territorios, constituye un esfuerzo meritorio y un avance importante que, aunque sea forzosamente genérico, supone un referente obligado para quienes este tema sea de interés (Estivill, 2003). En este contexto, es importante reconocer el aporte de la sociedad civil como actor contribuyente, mediante diversas estrategias y/o modelos de intervención social en la superación de estas exclusiones. Tal como lo señala Jordi Estivill,

la conciencia ciudadana de que la exclusión en todo el mundo nos afecta a todos y que todos en la medida de nuestras posibilidades podemos hacer algo, crece (...) Voz de una emergente y cada vez más consistente sociedad civil que se está convirtiendo en uno de los protagonistas internacionales, nacionales y locales de la acción contra la pobreza y la exclusión. Voz que se expresa con tonos y volúmenes muy distintos. Su composición y sus actuaciones también lo son. (2003, pp. 64-65)

Otros autores, como Ana Rizo, sostienen que la sociedad civil funciona como eje conector entre el Estado y el mercado. En este sentido, uno de los principios básicos y fundamentales para la actuación es la solidaridad (Rizo, 2006). A pesar de que las motivaciones religiosas o ideológicas aún siguen considerándose una fuente de ayuda social, actualmente, y con mayor frecuencia, aparecen organizaciones con criterios empresariales y relaciones de todo tipo con el sector público "que tratan no sólo de paliar los efectos negativos 
de la exclusión sino de encontrar las causas y tratar de erradicarlas" (Rizo, 2006, p. 15). Esta visión de la solidaridad se ha impuesto actualmente a los conceptos de "caridad" o "empatía de clase", y es entendida como un principio ético, transformador e integral.

\section{Conclusiones}

Al caracterizar el fenómeno de la migración y sus diversos aspectos en los asentamientos precarios, se observa que la intervención social debe ser particular y aplicada a cada contexto en el que se ejecute, ya que esto resulta una herramienta fundamental para contribuir en la superación de esta exclusión. Por ejemplo, contar con flexibilidad en un modelo de intervención permitiría generar estrategias adecuadas para la inclusión social de las personas con las que se trabaja. Esto ha supuesto todo un desafío para las OSC, como es el caso de Fundación Quiero mi Casa, que, desde un modelo tradicional, pasó a un modelo con un enfoque intercultural, en el que las intervenciones se adecuan a la realidad local, lo cual se dio a partir de la incorporación de migrantes en los asentamientos en los cuales trabaja.

En este sentido, el desafío de trabajar con personas que viven en asentamientos precarios y que, además, pertenecen a población extranjera, es relevante tanto para las políticas sociales y de superación de la pobreza, como para el trabajo de las organizaciones de la sociedad civil en general, ya que afronta el tener una metodología que incluya procesos y enfoques interculturales para sus respectivos marcos de acción. En este sentido, contar con un bagaje intercultural a la hora de intervenir ayudaría tanto a insertarse en un contexto poco explorado dentro de la política social nacional, como a optimizar el trabajo que se realiza, puesto que mejoraría las habilidades sociales de ambas partes, al promover la alteridad, disminuir las posibilidades de que el trabajo se ejecute con grados de etnocentrismo y, además, promover la participación, pues se reconoce al otro como un igual en derechos y deberes, y se parte del respeto y la valoración de la diversidad cultural (Stefoni, Stang y Riedeman, 2016). 
De esta forma, el trabajo con migrantes en asentamientos precarios cobra sentido cuando la participación de estos se hace activa en los procesos de intervención social, ya que, en ese espacio, se reconoce al otro en su diversidad y en su autonomía, sin importar su condición. Entender sus expectativas y sus deseos y circunstancias permite generar cambios a un modelo y/o estrategias de intervención acordes a su realidad actual, en los cuales la movilidad sea parte del proceso.

Como se ha visto, estas problemáticas generan múltiples desafíos, haciéndose necesaria la atención del Estado y la formulación de políticas públicas ad hoc, buscando soluciones eficientes y efectivas. Sin embargo, dadas las limitaciones del sector público ante la emergencia de lo que aquí denominamos subexclusiones, insertas a su vez en el problema de los asentamientos precarios, es menester la contribución de otros sectores.

En este espacio, nuevos actores comienzan a tomar un papel protagónico en llevar a cabo acciones que busquen disminuir las brechas de desigualdad existentes en Chile. El tercer sector de la sociedad civil organizada se configura como un actor social clave en la tarea de atender los acelerados cambios políticos, económicos, culturales, sociales y tecnológicos a los cuales el Estado, dado su limitada esfera de acción, no entrega soluciones satisfactorias. En la búsqueda de expresar opiniones, influir en las decisiones de gobierno, visibilizar demandas y entregar servicios, han contribuido de manera importante en la construcción de la sociedad y en el fortalecimiento de la democracia.

\section{Bibliografía}

Ander-Egg, E. (2017). Diccionario de Trabajo Social. Buenos Aires, Argentina: Ecro-Ilph.

Atkinson, R. \& Da Voudi, S. (2000). The Concept of Social Exclusion in the European Union: Context, Development and Possibilities. Journal of Common Market Studies, 38(3), 427-448.

Azuero, A. (2009). Capital Social e Inclusión Social: Algunos elementos para la política social en Colombia. Cuadernos de Administración. Universidad del Valle, 41. 
Fenómenos de exclusión social en asentamientos precarios: desafíos para las políticas públicas y las organizaciones de la sociedad civil, OSC / Fierro et al.

Bachiller, S. (2013). Teorías sobre la exclusión social: reflexiones acerca de su aplicabilidad en el análisis de los procesos de precariedad social que afectan a los recolectores informales de un basural municipal. Dossier Primer Encuentro Patagónico de Teoría Política.

Baeza, M. y Silva, G. (2009). Imaginarios sociales del Otro: el personaje del forastero en Chile (de 1845 a nuestros días). Concepción, Chile: Sociedad Hoy.

Bunges, M. (2008). Filosofía y Sociedad. México: Siglo XXI Editores S.A

Campos, A. (2012). Racialización, racialismo y racismo: un discernimiento necesario. La Habana: Universidad de La Habana.

Campos, K. (2017). Inmigración en Campamentos, Percepciones sobre la vida en Asentamientos Precarios de Copiapó. Chile: Revista Cis.

Casen. (2015). Encuesta de Caracterización Socioeconómica Nacional 2015. Santiago, Chile: Ministerio de Desarrollo Social y Familia.

Casen. (2015). Inmigrantes. Principales Resultados (Versión Extendida). Santiago, Chile: Ministerio de Desarrollo Social, Subsecretaría de Evaluación Social, 21 de diciembre de 2016.

Castel, R. (1997). La metamorfosis de la cuestión social. Buenos Aires: Paidós Estado y Sociedad.

Castells, M. (2001). La era de la información. Fin de milenio. Vol. 3. Madrid: Alianza Editorial.

CIS TECHO-Chile. (2017). Actualización catastro nacional de campamentos. Santiago, Chile: Fundación Techo-Chile.

CIS TECHO-Chile. (2015). Datos duros de una realidad mucho más dura: Encuesta Nacional de Campamentos 2015. Santiago, Chile: Fundación Techo-Chile.

Codoceo, F. y Muñoz, J. (2017). Fragmentación, asistencialismo e individualización de la responsabilidad: perspectivas territoriales concernientes a las percepciones ciudadanas de las políticas públicas en el contexto chileno de producción neoliberal. Sociedade e Estado, 32(2), 371-387.

Colomés, S. y Pavez, I. (2018). Derechos humanos y política migratoria. Discriminación arbitraria en el control de fronteras en Chile. Santiago, Chile: Polis.

Comisión de las Comunidades Europeas. (1992). Hacia una Europa de la Solidaridad. Intensificación de la lucha contra la exclusión social y la promoción de la integración. Bruselas: COM (92) 542 final. 
De Ramón, A. (2006). La población informal. Poblamiento de la periferia de Santiago de Chile, 1920-1970. En C. de Mattos, O. Figueroa, P. Bannen y D. Campos (Eds.), Santiago en EURE. Huellas de una metamorfosis metropolitana 1970/2000. Santiago: Instituto de Estudios Urbanos y Territoriales. Pontificia Universidad Católica de Chile.

Esping-Andersen, G. (1990). The Three Worlds of Welfare Capitalism. Estados Unidos: Princeton University Press.

Estivill, J. (2003). Panorama de la lucha contra la exclusión social. Conceptos y estrategias. Ginebra: Oficina Internacional del Trabajo.

Fonseca, M. y Fonseca, Z. (2004). Trabajo Social y migraciones intralatinoamericanas en Chile. De la xenofobia a la aceptación de lo diferente. San José, Costa Rica: XVIII Seminario Latinoamericano de Escuelas de Trabajo Social. La cuestión social y la formación profesional en Trabajo Social en el contexto de las nuevas relaciones de poder y la diversidad latinoamericana.

Fundación Quiero Mi Casa. (2019). Modelo y Manual de Intervención. Santiago de Chile.

Gómez, E., Fernando, D., Aponte, G. y Betancourt, L. (2014). Metodología para la revisión bibliográfica y la gestión de información de temas científicos, a través de su estructuración y sistematización. DYNA 81(184), 158-163.

Gordon, D., Adelman, L., Levitas, R. \& Pantazis, C. (2000). Poverty and Social Exclusion in Britain. York: Joseph Rowntree Foundation.

INE. (2018). Características de la inmigración internacional en Chile, Censo 2017. Santiago, Chile: Instituto Nacional de Estadísticas.

INE \& DEM. (2018). Estimación de Personas Extranjeras Residentes en Chile 31 de diciembre 2018. Santiago, Chile: Departamento de extranjería y migración.

INREDH. (2001). Diversidad ¿Sinónimo de Discriminación? Ecuador, Quito: Comunicaciones INREDH.

Iturra, D. (2016). De Haití a Chile: la formación de un enclave residencial en la periferia de Santiago. Santiago, Chile: Tesis para optar al grado de Magíster. Pontificia Universidad Católica de Chile.

Jiménez, M. (2008). Aproximación Teórica de la Exclusión Social: Complejidad e Imprecisión del Termino. Consecuencias para el Ámbito Educativo. Estudios Pedagógicos 34(1), 173-186. 
Fenómenos de exclusión social en asentamientos precarios: desafíos para las políticas públicas y las organizaciones de la sociedad civil, OSC / Fierro et al.

Larrañaga, O. (2010). Las nuevas politicas de protección social en perspectiva histórica. Programa de las Naciones Unidas para el Desarrollo. Recuperado de http://www.ministeriodesarrollosocial.gob.cl/btca/txtcompleto/Larranaga_nvaspolitprotecsocial.pdf

Lechner, N. (2002). Las sombras del mañana. La dimensión subjetiva de la política. Santiago, Chile: Colección Escafandra.

Lenoir, R. (1974). Les exclus: Un Française sur dix. Paris: Editions du Seuil.

Leyton, C. y Muñoz, G. (2016). Revisitando el concepto de exclusión social: su relevancia para las políticas contra la pobreza en América Latina. Revista del CLAD Reforma y Democracia, 65, 39-68.

Levitas, R., Pantazis, C., Fhamy, E., Gordon, D., Lloyd, E. \& Patsios, D. (2007). The Multi-Dimensional Analysis of Social Exclusion. Bristol: University of Bristol, Department for Communities and Local Government.

Marcuse, P. (2001). Enclaves Yes, Ghettoes, No: Segregation and the State. Cambridge, Massachusetts: International Seminar on Segregation in the City, Lincoln Institute of Land Policy.

Matus, T. (1999). Propuestas contemporáneas en Trabajo Social. Buenos Aires: Espacio editorial.

Ministerio de Desarrollo Social. (2015). CASEN Inmigrantes: principales resultados. Santiago, Chile: Subsecretaria de Evaluación Social, Ministerio de Desarrollo Social.

Ministerio de Planificación y Cooperación de Chile. (2012). Resultados de Encuesta de Caracterización Socioeconómica del año 1990 y 1996. Recuperado el 2 de agosto de 2019 de http:// observatorio.ministeriodesarrollosocial.gob.cl/casen_est_ pobreza.php

MINVU. (1985). Catastro de Marginalidad Habitacional. Chile: Ministerio de Vivienda y Urbanismo, Gobierno de Chile.

MINVU. (2007). Chile. Un siglo de políticas de vivienda y barrio. Chile: Ministerio de Vivienda y Urbanismo, Gobierno de Chile.

MINVU. (2009a). Catastro nacional de campamentos 2007. Chile: Ministerio de Vivienda y Urbanismo, Gobierno de Chile.

MINVU. (2009b). Déficit urbano-habitacional: una mirada integral a la calidad de vida y el hábitat residencial en Chile. Chile: Ministerio de Vivienda y Urbanismo, Gobierno de Chile.

MINVU. (25 de agosto de 2014). Ministra: La segregación residencial y marginalización de zonas completas de nuestras ciudades son inequidades urbanas que nos avergüenzan. Recupera- 
do el 06 de abril de 2017 de http://www.minvu.cl/opensite_det_20140825161943.aspx

MINVU. (8 de Marzo de 2018). Mi vivienda. Ministerio de Vivienda y Urbanismo: Recuperado de http://www.minvu.cl/opensite_20070311161723.aspx

Morales, E. F. (2018). Inmigrantes en campamentos en Chile: ¿mecanismo de integración o efecto de exclusión? INVI 33(94). Recuperado de https://scielo.conicyt.cl/scielo.php?script=sci_ arttext\&pid $=$ S071883582018000300161\#B10

Observatorio DESC: Derechos Económicos, Sociales y Culturales. (2013). Derecho a una vivienda digna. Recuperado el 2 de septiembre de 2019 en http://www.observatoridesc.org/ es/derecho-una-vivienda-adecuada

Olavarría, M. (2007). Conceptos básicos en el análisis de políticas públicas. Documento de trabajo $N^{\circ} 11$. Chile: INAP-Instituto de Asuntos Públicos. Departamento de Gobierno y Gestión Pública.

Organización de los Estados Americanos. (2011). Desigualdad e Inclusión Social en las Americas. 14 ensayos. Estados Unidos.

Palma, J. y Urzúa, R. (2005). Políticas contra la pobreza y ciudadanía social: el caso de Chile Solidario. Colección Políticas Sociales, № 12. Santiago, Chile: Departamento de Políticas Públicas, Instituto de Asuntos Públicos, Universidad de Chile.

Raczynski, D. y Serrano, C. (2005). Las políticas y estrategias de desarrollo social: aportes de los años 90 y desafíos futuros. En P. Meller (ed.), La paradoja aparante. Equidad y eficiencia: resolviendo el dilema. Santiago, Chile: Editorial Taurus.

Rivas, A. (2013). Campamentos: factores socioespaciales vinculados a su persistencia. (Actividad formativa equivalente para optar al grado de magíster en urbanismo). Santiago, Chile: Universidad de Chile. Recuperado de http://repositorio.uchile.cl/bitstream/handle/2250/116946/rivas_a.pdf;jsessioni $\mathrm{d}=7 \mathrm{FA} 80293 \mathrm{~B} 92 \mathrm{EAFA3070F77AD74878482}$ ? sequence $=1$

Rizo, A. (2006). ¿A qué llamamos exclusión social? Polis, Revista de la Universidad Bolivariana, 5(15). Universidad de Los Lagos, Santiago de Chile.

Rodríguez, A. (2012). Psicología Social Comunitaria: Vigencias y disonancias en los escenarios actuales. Comunicación en el II Simposio internacional en Psicología Social Comunitaria. Pereira, Risaralda: Universidad Abierta y a Distancia (UNAD).

Rodríguez, A., Pierri, L., Ana, R., Da Silva, M., Rossi, S. y Castro, I. (2013). Procesos subjetivos en la inclusión social. Una 
Fenómenos de exclusión social en asentamientos precarios: desafíos para las políticas públicas y las organizaciones de la sociedad civil, OSC / Fierro et al.

aproximación a través del método biográfico. Revista de Ciencias Sociales, 25(1), 96-111.

Rojas, N. y Silva, C. (2016). La Migración en Chile: Breve reporte y Caracterización. Madrid, España: Observatorio Iberoamericano sobre Movilidad Humana, Migraciones y Desarrollo.

Room, G. (2000). Trajectories of Social Exclusion: The Wider Context for the Third and First Worlds. In D. Gordon \& P. Townsend (eds.), Breadline Europe: The Measurement of Poverty. Bristol: Bristol Policy Press.

Stefoni, C. (2005). Migración en Chile. Chile: Colección Ideas.

Stefoni, C., Stang, F. y Riedemann, A. (2016). Educación e interculturalidad en Chile: Un marco para el análisis. Estudios internacionales (Santiago), 48(185), 153-182.

Winchester, L. (2008). La dimensión económica de la pobreza y precariedad urbana en las ciudades latinoamericanas. Implicaciones para las políticas del hábitat. EURE, 34(103), 27-47. 